\title{
Tecnologia da Informação e Comunicação na Formação Docente em Saúde: Relato de Experiência
}

\section{Information and Communication Technology in Teacher Training in Health: a Case Report}

Silvia Itzcovici Abensur Marcia Rodrigues Garcia Tamosauskas ${ }^{I I}$

\section{PALAVRAS-CHAVE \\ - Ensino. \\ - Aprendizagem. \\ - Criatividade. \\ - Tecnologia de Informação e Comunicação. \\ - Saúde}

Recebido em: 17/02/2010 Aprovado em: 30/06/2010 REVISTA BRASILEIRA DE EDUCAÇÃO MÉDICA $1 0 2 \longdiv { 3 5 ( 1 ) : 1 0 2 - 1 0 7 ; 2 0 1 1 }$
${ }^{I}$ Universidade de São Paulo, São Paulo, SP, Brasil

I" Faculdade de Medicina do ABC, Santo André, SP, Brasil.

\section{RESUMO}

Com o objetivo de desenvolver diversos olhares e possibilidades sobre ensino e aprendizagem na formação de pós-graduandos para a docência na Faculdade de Medicina da Universidade de São Paulo, em 2001 foi introduzida uma atividade de tecnologia de informação e comunicação. Esta atividade constitui uma oportunidade para os pós-graduandos aplicarem alguns recursos de informática e conhecerem o benefício da multidisciplinaridade, além de demonstrarem suas habilidades de criatividade e planejamento ao desenvolverem uma aula virtual. Ao final, os pós-graduandos avaliam a atividade com relação às características mais importantes e às dificuldades encontradas, emitindo comentários e sugestões. O objetivo deste trabalho é avaliar e relatar a experiência no período de 2006 a 2009, considerando-se as avaliações discentes (167) e os trabalhos desenvolvidos (193).

An information and communication technology activity was introduced in 2001 at the University of São Paulo, with the aim of developing various views and possibilities for training graduate students in teaching at the School of Medicine. The activity provided an opportunity for the graduate students to apply several key information technology resources and learn about the benefits of multidisciplinary approaches, in addition to testing their own creativity and planning skills for developing a virtual class. At the end, the graduate students evaluated the activity with comments and suggestions on its main characteristics and difficulties. The aim of the current article is to report on and assess the experience from 2006 to 2009, based on the evaluations by graduate students (167) and the resulting monographs (193). 


\section{INTRODUÇÃO}

As normas e determinações legais que regulamentam o desenvolvimento de programas de pós-graduação são originadas do Conselho Federal de Educação (CFE). De acordo com o artigo $5^{\circ}$ das Normas Complementares do Parecer 576/70, consta que "dos currículos dos cursos de pós-graduação em medicina deverão constar as disciplinas de Didática Especial, Pedagogia Médica, Problemas Brasileiros e o estudo das questões de deontologia pertinentes à área do curso respectivo" $^{\prime 1}$ (p. 2). Para dar cumprimento a estas exigências, as disciplinas de Pedagogia Médica e Didática Especial foram inseridas nos currículos dos alunos de pós-graduação da Faculdade de Medicina da Universidade de São Paulo (FMUSP) em 1974.

Em 1998, as duas disciplinas foram unificadas, contribuindo para integrar os conteúdos, evitando a superposição de temas. A disciplina Pedagogia Médica e Didática Especial é uma das disciplinas da FMUSP para capacitação didática e pedagógica do pós-graduando em programa senso estrito, com o objetivo de formar docentes qualificados. No conteúdo programático estão os elementos básicos da educação médica, planejamento e objetivos educacionais, teorias da aprendizagem, estrutura curricular e avaliação.

Para aperfeiçoá-la, foi introduzida, em 2001, uma atividade com o uso da tecnologia de informação e comunicação (TIC) com o objetivo de desenvolver diversos olhares e possibilidades sobre o ensino e aprendizagem na área da Saúde. O conteúdo aprovado para a disciplina criada em 1975 previa o "uso de computadores no ensino e na avaliação de aprendizagem. Simulação de problemas clínicos apresentados por computadores"11 (p. 11).

Esta inovação nas atividades da disciplina permite incorporar novas tecnologias em larga escala, e nos últimos trinta ou quarenta anos, em todos os setores de atividades, gerou, inegavelmente, profundas transformações econômicas e culturais na sociedade contemporânea ${ }^{2}$. Diante da entrada dos novos meios de tecnologia em nosso cotidiano, a educação não poderia caminhar longe deste contexto, pois, aproveitando estes mecanismos, ela abrange cada vez mais pessoas, em diferentes locais e com expectativas variadas daquilo que receberão, influenciando, muitas vezes, a facilidade e o prazer do indivíduo em aprender ${ }^{3}$.

O aprendizado com auxílio de recursos computacionais tem sido empregado com êxito tanto no Brasil como no exterior. Nesse cenário, softwares educacionais que utilizam multimídia vêm ajudando alunos e professores a tornarem o aprendizado mais fácil, rápido e eficiente ${ }^{2,4}$.

\section{OBJETIVO}

O objetivo deste trabalho é avaliar e relatar a experiência da introdução da atividade de tecnologia de informação e comunicação no período de 2006 a 2009 na disciplina destinada à preparação docente de pós-graduandos em programa senso estrito da FMUSP.

\section{ATIVIDADE}

Em 2001, a atividade constava da exibição de uma hora de aplicativos computacionais utilizados para pesquisa, educação ou prática profissional em Saúde. Alguns dos exemplos de aplicação educacional apresentados foram os seguintes temas: Determinantes da ultrafiltração glomerular ${ }^{5-7}$; Distúrbios do metabolismo da água ${ }^{5}$; Desidratações ${ }^{5}$; e Imunodeficiências. Estes são alguns dos aplicativos utilizados na disciplina Bases Fisiológicas da Prática Médica, oferecida aos alunos do segundo ano de graduação do curso de Medicina da FMUSP.

Para a prática profissional foram apresentados como exemplos os temas: Índices de classificação de gravidade das doenças; Adequação em diálise peritoneal ${ }^{8}$; e Cálculo do índice litiogênico. E como exemplos de animações didáticas, os temas: Mecanismo de ação da ciclosporina; Mecanismo de transporte de solutos e água através da membrana peritoneal; e Roteiro para o tratamento da anemia.

A partir do primeiro semestre de 2005, a duração desta atividade foi ampliada para quatro horas (duas horas oferecidas em dois encontros), possibilitando incluir uma atividade prática numa sala com 50 computadores em rede. No primeiro semestre de 2005, só havia um exercício prático, no segundo semestre desse ano havia dois exercícios, e, a partir de 2006, três exercícios faziam parte da atividade.

Atualmente, no início do primeiro encontro continuam a ser apresentados os exemplos dos aplicativos. Com os recursos disponíveis da sala, também é permitida sua exploração simultânea pelos alunos durante a apresentação pelo professor A execução das instruções pelo professor é visualizada num telão e repetida sempre que solicitada.

Na segunda parte, de acordo com um roteiro, são abordados alguns recursos de informática sob a forma de três exercícios: construção de hiperlinks no Power Point Microsoft, cálculos simples e criação de barras de rolagem no Excel Microsoft. O primeiro exemplo, elaborado no Power Point, é de uma questão de múltipla escolha com cinco opções. Com o uso de hiperlinks, cada opção pressionada direciona o aplicativo para a respectiva resposta/comentário, simulando um estudo dirigido. O segundo exemplo é a exibição do comportamento de uma equação de segundo grau no Excel, onde o valor de cada um dos três parâme- 
tros é escolhido pelo usuário pela movimentação da respectiva barra de rolagem, sendo que o gráfico ajusta instantaneamente o formato da curva aos valores assumidos. E o terceiro exemplo é o cálculo do índice de massa corpórea9: escolhendo-se o valor do peso de uma pessoa numa barra de rolagem, o gráfico do índice da massa corpórea é instantaneamente atualizado.

Ao final da atividade do primeiro encontro, os alunos preenchem um questionário semiestruturado, de adesão voluntária, sobre o que consideraram mais importante na atividade, suas dificuldades, com comentários e sugestões.

No segundo encontro, os alunos iniciam a elaboração do trabalho final, além de utilizarem o momento para discussão e esclarecimento de dúvidas. A inserção do trabalho final ocorreu a partir de 2006. Como atividade final, compete ao aluno elaborar uma aula virtual, ou seja, construir um aplicativo (individualmente ou em dupla) cujo tema esteja relacionado com sua área de atuação, utilizando os recursos de informática descritos no primeiro encontro. Também como premissa deste trabalho, é solicitado que o aplicativo elaborado seja de utilização intuitiva, isto é, o usuário não necessita da presença do autor e nem de instruções além do próprio aplicativo. Além disso, o produto final deverá ser um fator motivador de aprendizado, contribuindo para a aquisição de conhecimento pelo usuário, numa experiência significativa e efetiva.

\section{RESULTADOS}

Durante o período analisado (primeiro semestre/2006, segundo semestre/2006, primeiro semestre/2007, segundo semestre/2007, primeiro semestre/2008 e primeiro semestre/2009), 234 pós-graduandos desenvolveram, individualmente ou em grupo, 193 aplicativos (aulas virtuais) com temas relacionados às respectivas formações (30\% de Medicina, $16 \%$ de Fisioterapia, $7 \%$ de Fonoaudiologia e $47 \%$ de outras áreas).

A variedade de assuntos e a criatividade na elaboração dos trabalhos foram grandes, demonstrando o envolvimento dos participantes na atividade. Em relação ao software empregado no trabalho, $66 \%$ usaram apenas o Power Point e $7 \%$ apenas o Excel, enquanto $27 \%$ aproveitaram os recursos dos dois softwares, criando um link entre eles.

Alguns dos títulos dos trabalhos desenvolvidos pelos pós-graduandos são: Estudo Dirigido: Processamento Auditivo; Doença Periodontal; SUS: Sistema Único de Saúde; Promoção de Saúde e Segurança no Trabalho; Escala Glasgow Coma; Avaliação da Dieta; Insônia de Lactentes; Estadiamento de Câncer de Boca; Efeitos das Drogas Psicotrópicas; Diagnóstico do Esôfago de Barrett; Doença de Chagas; Fisiologia e Fisioterapia Respiratória; Miniatlas de Citogenética; Princípios da Bioética; Manual Virtual de Audição.
Este último trabalho foi aperfeiçoado e apresentado no Congresso Brasileiro de Fonoaudiologia (2008) pela pós-graduanda autora ${ }^{10}$. Consta de um instrumento educacional interativo dirigido ao público leigo com enfoque na orientação e acesso à informação sobre a audição na criança com relação a atenção auditiva, importância, desenvolvimento e alterações.

Para finalizar o processo de aprendizagem, após a entrega do trabalho, os pós-graduandos recebem uma análise pormenorizada sobre o projeto desenvolvido, avaliando apresentação visual, organização, originalidade, clareza na navegação, proposta do aplicativo, quantidade e qualidade do conteúdo, recursos utilizados, funcionamento e estímulo ao usuário.

As categorias mais citadas nas 167 avaliações preenchidas pelos pós-graduandos (71\%) em relação ao item dificuldade foram relacionadas ao acompanhamento e construção dos exemplos no Excel Microsoft (31\%), além de problemas com os equipamentos da sala de aula, como microfone, falhas dos equipamentos e configuração dos micros. Houve algumas citações de não acompanhamento completo da aula, das instruções, da sequência de exemplo devido à falta de prática, nova linguagem, novas informações e raciocínios.

Foram citados como aspectos mais importantes da atividade: aprender e conhecer recursos e dicas de informática como uma ferramenta útil de aplicação imediata (51\%) e o fato de as apresentações dos exemplos tornarem amigável o contato com a informática. Alguns pretendem utilizar os recursos em suas atividades docentes, pois consideram que as aulas se tornam mais dinâmicas e se incentiva o processo ensino-aprendizagem, enquanto outros relatam que a atividade despertou a vontade de conhecer mais recursos de informática.

A incorporação do roteiro de atividade, em 2006, surgiu para atender às sugestões dos alunos em suas avaliações, constituindo um recurso adicional para o acompanhamento e fixação do conteúdo da atividade.

Sugestões frequentes foram aumento da carga horária $(28 \%)$, presença de monitores $(15 \%)$ e divisão em pequenos grupos (4\%), que será atendida para turmas posteriores com a divisão da classe em três subgrupos. Esta modificação na atividade talvez atenda, em parte, à solicitação de incluir vários níveis de conhecimento, já que a classe heterogênea limita o avanço da atividade.

Sugestões mais amplas, como incluir outros assuntos relacionados - elaboração de animação, estatística, edição de imagens, cores e fontes para melhor visualização de apresentações -, são mais difíceis de incorporar ao curso, devido à carga horária.

De modo geral, os pós-graduandos classificaram a atividade como excelente (29\%), interativa e ilustrada (11\%), es- 
truturada e organizada (11\%), e o assunto, interessante, transmitido de forma clara e com boa didática (11\%). Quanto aos recursos de informática explorados, consideram que facilitam o trabalho do docente, além de representarem uma forma excelente, interessante (15\%), enriquecedora, estimulante e eficiente de introduzir um assunto em aula.

\section{CONCLUSÕES}

Nesta atividade, os pós-graduandos da área de Saúde têm a oportunidade de aprender alguns recursos de informática e conhecer o benefício da multidisciplinaridade. Além disso, desenvolvem a criatividade e o planejamento ao projetarem um aplicativo de pequeno grau de complexidade de um assunto sobre o qual têm domínio e grande conhecimento. Estas duas competências são muito importantes para o aprendizado como um todo e permitem ampliar a utilização destas habilidades para outras áreas do conhecimento ${ }^{11}$. É visível a dificuldade da escolha do tema e a seleção crítica de informações e o consequente desafio de obedecer a todas as premissas exigidas para a finalização do trabalho.

Durante o desenvolvimento desta atividade, os participantes percebem a importância da revisão, da autocrítica, da apresentação visual e da organização, pois num aplicativo mal apresentado o conteúdo não é captado de forma adequada. Se a atividade multimídia é cuidadosamente desenhada, os usuários podem acompanhá-la em seu próprio ritmo, acessar facilmente a informação e se engajar de forma independente num aprendizado por descoberta ${ }^{12}$.

A elaboração de estudos dirigidos está associada ao conceito de organização em hipertexto, que favorece uma leitura (exploração ou navegação) não linear baseada em indexações, conexões entre ideias e conceitos articulados por meio de links (nós e ligações). Desta forma, ao se clicar sobre uma palavra, imagem ou frase definida como um nó de um hipertexto, encontra-se uma nova situação, evento ou outros textos relacionados ${ }^{13}$. Cada usuário do ambiente de estudo tem a oportunidade de percorrer caminhos distintos entre as informações, textos, hipertextos e imagens, que representam espaços de referência e interação que podem ser visitados, explorados, trabalhados, não caracterizando local de visita obrigatória $^{13}$.

A elaboração dos aplicativos permite a atuação dos participantes na perspectiva de docentes e de aprendizes ativos, críticos e criativos. Este processo estimula a liberdade de criação e o exercício do planejamento e permite a reflexão sobre a experiência de ensinar e aprender. $\mathrm{O}$ verdadeiro ensino é aquele que contribui com a formação da personalidade do estudante, encorajando-o a adquirir novos hábitos e atitudes, despertando sua curiosidade e induzindo-o a pensar sobre o assunto em estudo ${ }^{14}$. Dessa forma, "o exercício da docência nunca é estático e permanente; é sempre processo, é mudança, é movimento, é arte; são novas caras, novas experiências, novo contexto, novo tempo, novo lugar, novas informações, novos sentimentos, novas interações"15 (p. 15).

As vantagens da utilização dos aplicativos de simulação computacional incluem, além da privacidade na utilização, controle local sobre o tópico, o tempo, o lugar, a velocidade da instrução. Permitem interatividade com retorno imediato, e é justamente quando o aluno toma decisões na interação com o mundo virtual que ocorre o aprendizado. Os programas de simulação, por serem interativos, forçam o envolvimento do usuário, providenciando informações selecionadas que dependem dos desejos e necessidades do usuário, promovendo o aprendizado.

Aprende-se a conhecer, aprendendo a fazer e a refletir sobre esse fazer ${ }^{16}$. Qualquer pessoa aprende melhor fazendo, atuando, descobrindo e não por meio de pura memorização do conhecimento. $\mathrm{O}$ computador permite a aprendizagem autônoma, em que o aluno vai poder experimentar uma aprendizagem mais natural, que, segundo Schank e Cleary ${ }^{17}$, é composta por cinco formas de aprendizagem: aprender fazendo, aprendizado incidental, aprendizado por reflexão, ensino baseado em caso e aprendizado por exploração.

Os aprendizes possuem diferentes estilos de preferência no recebimento das informações ${ }^{12,18}$, e os programas computacionais podem ser confeccionados para se adaptar a estilos particulares de aprendizado contendo uma grande quantidade de informação. Ainda como vantagem, tem-se o desenvolvimento da habilidade em resolver problemas e enfrentar novas situações, estimulando a criatividade, a curiosidade, o espírito crítico e o espírito científico. Também é possível simular diversas situações, sem perigo ou incômodo aos pacientes ${ }^{19}$.

Estes programas podem superar as aulas práticas tradicionais com animais de laboratório, já que permitem simular situações cujas variáveis nem sempre são fáceis de alterar ou não podem ser modificadas em conjunto com outras. Além disso, existem objeções quanto à utilização de animais para ensino e evidentes limitações logísticas para a administração de aulas práticas que envolvam experimentos complexos a dezenas ou centenas de estudantes.

As novas tecnologias só terão sentido a partir de uma mudança da postura pedagógica do professor e com um repensar deste sobre sua própria prática, ao conceber que existem outras maneiras de explorar e representar o mundo ${ }^{20}$. As tecnologias de comunicação não substituem o professor, mas modificam algumas de suas funções ${ }^{21}$. 
Estes aplicativos constituem material didático adicional ou instrumento facilitador de aprendizado ${ }^{22}$, já que não substituem o professor ou o livro-texto, mas são encarados como recurso complementar para enriquecer e favorecer o processo educativo. As tecnologias são só apoio, meios, mas elas nos permitem realizar atividades de aprendizagem de formas muito diferentes das convencionais ${ }^{21}$. E o material sob a forma de CD-ROM possibilita a utilização off-line (independente de conexão com a internet). Desta maneira, a internet e estes recursos podem proporcionar acesso aos conhecimentos, permitindo ao professor abandonar o papel de transmissor de informação para desempenhar o de guia e conselheiro para o estudante ${ }^{22}$.

O conhecimento destes recursos didáticos não trará a aptidão necessária à autonomia tecnológica, mas esta experiência mostra como a multidisciplinaridade pode contribuir para o desenvolvimento de aplicativos de maior nível de complexidade, pois os estudantes percebem a necessidade do trabalho colaborativo com profissionais de distintas áreas do conhecimento, como, por exemplo, educação, informática, modelagem matemática de processos e saúde.

O ensino na área da Saúde precisa de profissionais tecnicamente competentes na área de atuação e didática, mas que, ao mesmo tempo, estejam conectados com novas linguagens e metodologias de aquisição de conhecimento e sejam capazes de enfrentar o desafio de um trabalho interdisciplinar e multiprofissional.

\section{REFERÊNCIAS}

1. Didática Especial e Pedagogia Médica no curso de Pós-Graduação da Faculdade de Medicina da Universidade de São Paulo. São Paulo: USP; 1993. (Documento CEDEM, 2)

2. Miranda CEA. Pesquisa em educação e imagens, novas tecnologias e a busca pela interlocução. Com Ciência [periódico na internet]. 2009[acesso em 20 ago. 2009];(110):[cerca de 7 p.]. Disponível em: http:/ /www.comciencia.br/co mciencia $/$ ?section $=8 \&$ edicao $=48 \& i d=598$

3. Caldas RF. Novas tecnologias para uma nova educação [online]. [acesso em 7 set. 2009]. Disponível em: http:// cdchaves.sites.uol.com.br/novas_tecnologias.htm

4. Assis WS, Bittencourt TN, Noronha MAM. Desenvolvimento de Recursos Multimídia para o Ensino de Engenharia de Estruturas [online]. 2002 [acesso em 7 set. 2009]. Disponível em: http://www.lmc.ep.usp.br/pesquisas/ TecEdu/artigos/artigo_Ibracon.pdf

5. Renke HG, Rose BD, Zatz R. Fisiopatologia Renal. São Paulo: Atheneu; 2000. v.2

6. Abensur SI, Zatz R. Dispositivo suplementar para o ensino de Fisiologia Renal. Anais do 26 Congresso Nacional de Matemática Aplicada e Computacional (CNMAC); São José do Rio Preto (SP); 2003.

7. Abensur SI, Zatz R. Simulação Virtual em Sala de Aula. Anais do $43^{\circ}$ Congresso Brasileiro de Educação Médica; Natal (RN); 2005.

8. Abensur SI, Abensur H. Calculador da Dose de Diálise ofertada aos pacientes em Programa de Diálise Peritoneal. Anais do 26 Congresso Nacional de Matemática Aplicada e Computacional (CNMAC); São José do Rio Preto (SP); 2003.

9. Livingston EH, Lee S. Body surface area prediction in normal-weight and obese patients. Am J Physiol Endocrinol Metab. 2001;281:E586-E591.

10. Horacio CH, Goffi-Gomez MVS, Abensur SI. Manual Virtual de Compreensão da Audição: para o Público Leigo. [Trabalho apresentado no $16^{\circ}$ Congresso Brasileiro de Fonoaudiologia. Campos do Jordão, SP; 2008.]

11. Rodrigues G. Que venham os robôs. Profissão Mestre. 2008; p.20-22.

12. Nascimento ACA. Princípios de design na elaboração de material multimídia para a WEB. Projeto RIVED [online]. 2000 [acesso em 19 mar. 2009] Brasília:MEC. Disponível em: http:/ /rived.mec.gov.br/instrumentos/design_pedagogico.pdf

13. Almeida MEB. Educação a distância na internet: abordagens e contribuições dos ambientes digitais de aprendizagem. Educ Pesqui. 2003;29(2):327-40.

14. Beraldo WT, Alvarenga GP. Non-directive method for teaching physiology. Physiologist. 1983; 26(4):229-30.

15. Cunha MI. Inovações pedagógicas: o desafio da reconfiguração de saberes na docência universitária. Cad Pedagogia Universit. 2008;6.

16. Almeida MEB. Tecnologia de informação e comunicação na escola: novos horizontes na produção escrita [online]. [acesso em 20 ago. 2009]. Disponível em: http:/ /www.iar. unicamp.br/disciplinas/mm_educacao/doc/TI\%20e\%20 CM\%20na\%20escola.doc

17. Schank RC, Cleary C. Engines for education. New Jersey: Lawrence Erlbaum Associates;1995.

18. Friedman CP, France CL, Drossman DD. A randomized comparison of alternative formats for clinical simulations. Med. Decis. Making. 1991;11(4):265-72.

19. Barnett GO. Information technology and medical education. J Am Med Inform Assoc. 1995; 2(5):285-91.

20. Oliveira FCF. As novas tecnologias na Educação Superior [online]. [acesso em 18 mar. 2009]. Disponível em: http:/ / www.unirverde.org/FARO/Apostila/apostila_Novas_ tecnologias.doc 
21. Moran JM. Mudanças profundas e urgentes na educação [online]. [acesso em 7 set. 2009]. Disponível em: http:// www.eca.usp.br/prof/moran/profundas.htm

22. Amem BMV, Nunes LC. Tecnologias de Informação e Comunicação: Contribuições para o Processo Interdisciplinar no Ensino Superior. Rev Bras Educ Med. 2006;30(3):171-80.

\section{CONTRIBUIÇÃO DOS AUTORES}

Silvia Itzcovici Abensur participou do desenvolvimento deste estudo, da interpretação dos dados, da redação da primeira versão do artigo. Marcia Rodrigues Garcia Tamosauskas participou de forma suficiente da concepção e desenvolvimento do estudo e da redação do texto e suas revisões.
CONFLITO DE INTERESSES

Declarou não haver.

\section{ENDEREÇO PARA CORRESPONDÊNCIA}

Silvia Itzcovici Abensur

Centro de Desenvolvimento de Educação Médica

Faculdade de Medicina da Universidade de São Paulo

Av. Macuco, 58 - ap. 11

Moema - São Paulo

CEP 04523-000 